\title{
DILEMA DO PRISIONEIRO NA ANÁLISE EXPERIMENTAL DO COMPORTAMENTO: UMA REVISÃO SISTEMÁTICA DA LITERATURA
}

PRISONER'S DILEMMA IN THE EXPERIMENTAL ANALYSIS OF BEHAVIOR: A SYSTEMATIC REVIEW

\author{
DAFNE PAVANELLI FIDELIS \\ UNIVERSIDADE FEDERAL DE SÃo CARLOS, BRASIL \\ PEDRO BORDINI FALEIROS \\ UnIVERSIDADE METODISTA DE PIRACICABA, BRASIL
}

\begin{abstract}
RESUMO
O Dilema do Prisioneiro é um jogo desenvolvido pela Teoria dos Jogos e estabelece um conflito entre "interesses" comuns e individuais. O objetivo do estudo foi realizar uma revisão sistemática de experimentos que utilizaram como procedimento o Dilema do Prisioneiro pautado na Análise Experimental do Comportamento, publicados em periódicos científicos. O procedimento foi composto de cinco etapas: (1) Definição das palavras-chave e seleção dos portais e indexadores de artigos científicos; (2) Busca e seleção dos artigos nos portais e indexadores; (3) Busca e seleção dos artigos em periódicos voltados à publicação em Análise do Comportamento; (4) Seleção das referências encontradas nos artigos lidos; (5) Definição e classificação dos temas. Foram identificados e selecionados 23 artigos. Dois roteiros de estudos foram utilizados para sistematizar as informações. Os estudos que utilizaram o jogo à luz do modelo de seleção do comportamento pelas consequências manipularam variáveis relacionadas às relações sociais, autocontrole e metacontingências. A relação entre o Dilema do Prisioneiro e a análise experimental do comportamento tem sido acentuada nas últimas décadas, o que sugere um aumento e continuidade de estudos nessa área.
\end{abstract}

Palavras-chave: dilema do prisioneiro, análise experimental do comportamento, autocontrole, metacontingências, comportamento social.

\begin{abstract}
The Prisoner's Dilemma is a game that derived from game theory. It establishes a conflict between individual and collective interests. The purpose of this systematic review was to identify and analyze studies that employed the Prisoner`s Dilemma, based on behavior analysis, and were published in scientific journals. The systematic review included five steps: (1) define keywords and select scientific indexing databases, (2) search and select articles in the databases, (3) search and select articles in specialized behavior analysis journals, (4) search the bibliographies in the identified articles, and (5) define and classify themes. At the end of the search, 23 articles were selected for the review. Two scripts were used to systematize the information. Studies that employed the Prisoner's Dilemma, based on the model of selecting behavior according to consequences, manipulated variables that were related to social relations, selfcontrol, and metacontingencies. The relationship between the Prisoner's Dilemma and the experimental analysis of behavior has been accentuated in the last decades, suggesting an increase in and continuity of studies in this area.

Keywords: Prisoner's Dilemma game, experimental analysis of behavior, self-control, metacontingencies, social behavior.
\end{abstract}

Este trabalho é produto da iniciação científica da primeira autora, que teve financiamento da FAPIC - Fundação de Apoio à Pesquisa de Iniciação Científica Unimep/ Protocolo CONSEPE 13213/ 2013-2014. Correspondência para Dafne Fidelis (dafnepfidelis@ hotmail.com) ou Pedro Faleiros (pbfaleiros@gmail.com). 
O Dilema do Prisioneiro foi definido inicialmente pela Teoria dos Jogos por Merrill Flood e Melvin Dresher, no ano de 1950, e posteriormente nomeado por Albert Tucker (Poundstone, 1992). Ele é descrito por uma situação que envolve dois infratores que são presos pela polícia e interrogados em celas separadas, sem nenhuma possibilidade de comunicação entre si. Esses infratores realmente cometeram um crime, porém não há provas. Os interrogadores apresentam aos dois presos o seguinte dilema (Figura 1): se os dois delatam, ambos receberão uma pequena pena (e.g., um ano). Se ambos cooperarem entre si e não delatarem, os dois infratores ficarão presos por um período intermediário (e.g., três anos). Se um delatar, mas o outro não, o delator fará um trato com a polícia e ficará livre, enquanto o outro terá um tempo de pena maior do que as outras mencionadas (e.g., cinco anos).

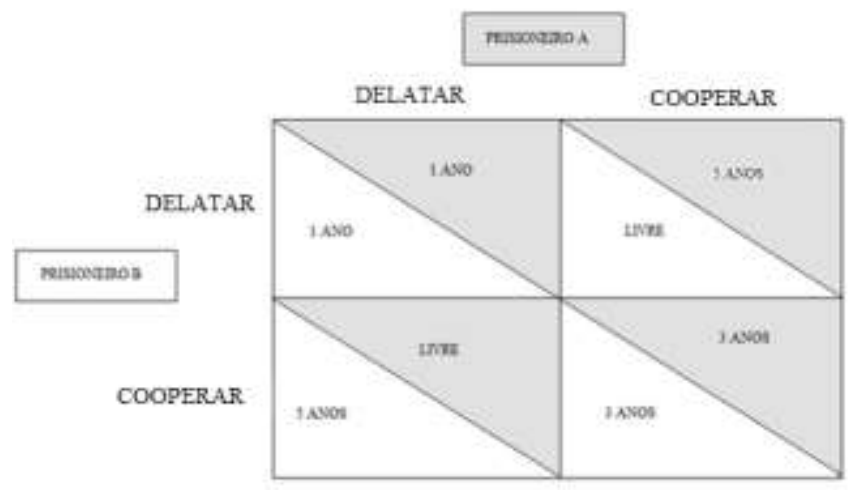

Figura 1. Tempo de pena para os Prisioneiros A e B, a depender das escolhas feitas por ambos no Dilema do Prisioneiro.

Dawes (1980), Kollock (1998), e Kopelman, Weber e Messick (2002) revisaram as variáveis manipuladas em experimentos que utilizaram o Dilema do Prisioneiro, com enfoque na Psicologia Social e Cognitiva. Tais estudos podem ser classificados como revisões narrativas de literatura por: (1) não possuir critérios explícitos e sistemáticos; (2) não realizar técnicas de busca metodologicamente estruturadas; (3) não empregar critérios para a interrupção da coleta de dados e (4) não apresentar um método sistemático de como a pesquisa foi realizada. ${ }^{1}$

Com base em termos e definições cognitivas, Dawes (1980) apresentou as seguintes variáveis manipuladas nos experimentos por ele identificados: grau de envolvimento dos sujeitos, que se refere ao nível de comprometimento do indivíduo nos jogos de dilemas sociais, a depender da quantidade de dinheiro e/ou recompensas obtidas; possibilidade de comunicação; tamanho do grupo; manifestação pública da escolha dos sujeitos versus escolhas privadas; expectativa sobre o comportamento dos outros, que se refere ao efeito da expectativa do jogador em relação à escolha do outro jogador, entre cooperar e competir.

\footnotetext{
${ }^{1}$ http://www.ip.usp.br/portal/images/biblioteca/revisao.pdf
}

O estudo reportado por Kollock (1998), também com base na Teoria Social Cognitiva, teve como objetivo apresentar e discutir variáveis em experimentos voltados a investigar e solucionar dilemas sociais, dentre eles o Dilema do Prisioneiro: (1) Soluções motivadas: quando os atores não são completamente egoístas e, portanto, consideram os resultados de seus parceiros, como por exemplo a comunicação entre eles; (2) Solução de estratégias: envolve a alteração no comportamento dos jogadores no decorrer do jogo, como reciprocidade e acordo mútuo; (3) Soluções estruturais: envolvem alterar as regras ao longo do jogo, como valor dos ganhos e tamanho do grupo.

No artigo de Kopelman, Weber e Messick (2002), os experimentos apresentados foram agrupados em diferenças individuais, que envolvem motivos sociais (características intrínsecas dos jogadores, como cooperativo ou competitivo, que influenciam a forma como vão distribuir os ganhos obtidos para si próprio e para o outro) e gênero, e em fatores situacionais, sendo esses perceptuais e de estrutura da tarefa. Fatores perceptuais referem-se ao nível de percepção do jogador (se o jogo como um todo foi interpretado com cooperativo ou competitivo), influenciando a forma de como serão feitas as escolhas. Estrutura da tarefa envolve o poder e status exercidos entre os jogadores, tamanho do grupo, comunicação e incerteza dos ganhos.

Outra revisão de literatura narrativa sobre o Dilema do Prisioneiro, mas com experimentos pautados na Análise Experimental do Comportamento, foi realizada por Rachlin, Brown e Baker (2001). As variáveis manipuladas nos estudos apresentados foram: história de reforçamento; atraso e magnitude do reforço; instruções; probabilidade de reciprocidade entre os jogadores e presença ou ausência de outro participante como estímulo discriminativo.

As revisões de Dawes (1980), Kollock (1998), Kopelman et al. (2002), e Rachlin et al. (2001) não utilizaram métodos claramente formulados para encontrar, selecionar e analisar os resultados de experimentos relevantes para a área pesquisada, conforme exige uma revisão sistemática de literatura. Além disso, na presente pesquisa, sobre estudos que relacionam a Análise Experimental do Comportamento e o Dilema do Prisioneiro, não foi encontrada revisão sistemática de literatura.

Segundo a American Psychological Association (2010), uma revisão sistemática deve conter a descrição de critérios para seleção dos artigos (inclusão e exclusão) e de como a revisão foi realizada, de modo que a replicação seja possível, apresentando os principais termos usados na busca (e o idioma em que foram usados); bancos de dados pesquisados e período pesquisado. Alarcão, Cardoso e Celorico (2010) defenderam que uma revisão sistemática de literatura permite que os leitores possam ter uma visão abrangente e profunda sobre os conhecimentos produzidos em uma determinada área. O objetivo do presente estudo foi realizar uma revisão sistemática de experimentos que utilizaram como procedimento o Dilema do Prisioneiro, pautados na Análise Experimental do Comportamento, publicados em periódicos científicos. 


\section{MÉTODO}

\section{Fontes de busca}

Para a busca dos artigos, foram utilizados os seguintes portais e indexadores: Google Acadêmico, Periódicos CAPES, BVS-Psi, Scielo, Pepsic e também os periódicos da Association for Behavior Analysis International $(\mathrm{ABAI})^{2}$. $\mathrm{O}$ critério de escolha dos indexadores foi definido pelos mesmos estarem disponíveis na internet e com acesso gratuito.

Para a sistematização das informações referentes ao processo de revisão sistemática foi utilizada a ferramenta computacional START® versão $2.0^{3}$. O objetivo desse programa é auxiliar o processo de Revisão Sistemática em todas as suas etapas: planejamento, execução e análise dos resultados (Zamboni, Thommazo, Hernandes, \& Fabbri, 2010).

\section{Procedimento}

O procedimento foi delineado em cinco etapas descritas a seguir.

Primeira etapa: Definição das palavras-chave. Inicialmente foram utilizadas as seguintes palavras-chave para a busca online dos artigos: "Behavior Analysis" e "Prisoner's Dilemma". O uso das palavras em inglês foi definido por haver uma maior quantidade de artigos publicados nessa língua e pelo fato de que mesmo que o artigo seja publicado em outra língua, os periódicos científicos têm exigido, pelo menos, título e resumos na língua inglesa.

\section{Segunda etapa: Busca e seleção dos artigos nos portais e indexadores. \\ As palavras "Behavior Analysis" e "Prisoner's} Dilemma" sempre foram inseridas juntas nos campos de busca como "palavras exatas" nos portais e indexadores. Cabe salientar que essas duas palavras foram colocadas entre parênteses, para que a busca fosse exatamente feita com base nas palavras digitadas no campo, uma vez que cada item de busca continha duas palavras. Além disso, em todos os indexadores e periódicos pesquisados, a busca era feita da forma "completa", considerando, o título, autor, resumo e palavras-chaves. No presente estudo não foi definido um determinado período de tempo para a busca dos artigos, visto que a hipótese era que a quantidade de artigos publicados ao longo dos anos não seria tão expressiva.

Após as referências serem encontradas, elas eram importadas para o programa START®. Com as referências salvas no programa, o próximo passo era selecionar a opção "duplicação automática das referências", de modo que elas não fossem contabilizadas mais de uma vez. Caso as referências importadas no programa fossem as mesmas, mas apresentassem títulos em línguas diferentes, uma nova

\footnotetext{
${ }^{2}$ https://www.abainternational.org/journals.aspx

${ }^{3}$ A ferramenta computacional START® versão 2.0 está disponível online no endereço

http://lapes.dc.ufscar.br/tools/start_tool
}

leitura dessas referências era realizada para que as mesmas não fossem contabilizadas mais que uma vez.

Após as referências terem sido importadas e salvas no programa, o próximo passo foi a leitura dos resumos e a seleção daqueles que apresentavam os critérios de uma pesquisa experimental. $\mathrm{O}$ artigo era selecionado se o método tal como descrito no abstract informasse sobre a participação de sujeitos experimentais (humanos ou não) ou "agentes computacionais" (jogadores simulados) e se havia manipulação de variáveis.

Após a seleção dos artigos experimentais, foi realizada uma nova leitura dos abstracts. O critério dessa vez foi se o abstract apresentava termos empregados pela análise experimental do comportamento. Tais termos não foram definidos previamente. Os termos identificados como analítico-comportamentais nos artigos selecionados nessa etapa foram: reforçamento, metacontingências, contingência cultural, comportamento governado por regras, comportamento governado por contingências, comportamento verbal e não verbal, efeito das consequências, estimulação antecedente ou consequente, punição, comportamento cooperativo, lei da igualação, paradigma de emparelhamento probabilístico, taxas de comportamento, condicionamento respondente, atraso no reforço, consequências de longo prazo, comportamento aversivo e reforçamento interpessoal.

O próximo passo foi a seleção dos artigos que, além de abranger os princípios da Análise do Comportamento e ser experimental, também estavam relacionados com o Dilema do Prisioneiro. Ao acessar o artigo completo no programa Adobe Reader $\AA$, na ferramenta "Localizar" eram inseridas as palavras "Prisoner's Dilemma". Caso essas palavras fossem encontradas em qualquer lugar no corpo do texto, com exceção da seção de referências, o mesmo era selecionado.

Terceira etapa: Busca e seleção dos artigos em periódicos de Análise do Comportamento.

Para cada um dos periódicos encontrados no site da ABAI foram realizadas buscas com a palavra-chave "Prisoner's Dilemma". Os artigos encontrados nessa etapa também foram importados para o programa Start ${ }^{\circledR}$ e foi realizado o mesmo processo de seleção feito com os artigos encontrados nos portais indexadores.

A seleção dos artigos considerando os critérios "Pesquisa Experimental" e "Dilema do Prisioneiro" foram os mesmos utilizados na seleção nos portais e indexadores. No entanto, não foi necessário utilizar o critério "Análise do Comportamento" na terceira etapa, pelo fato dos periódicos estarem disponíveis no site ABAI.

\section{Quarta etapa: Seleção das referências.}

$\mathrm{Na}$ quarta etapa, novamente foi selecionado o ícone "duplicação" no Start $\AA$, de modo que o mesmo artigo não fosse contabilizado mais de uma vez. Em posse dos artigos até então selecionados, com objetivo de ampliar a busca, foi feita a leitura dos títulos apresentados nas seções de referências de cada artigo, e foram selecionadas aquelas nas quais fossem encontradas as seguintes palavras na língua inglesa: Dilema do 
Prisioneiro, cooperação, cooperativo, delação, delatar, competição, competitivo, autocontrole, altruísmo e egoísmo. As palavras utilizadas para essa busca foram definidas previamente, pelo fato de estarem relacionadas ao Dilema do Prisioneiro.

Nesta etapa, os critérios para definir se o artigo era experimental, pautado na Análise do Comportamento e que empregasse o Dilema do Prisioneiro, foram idênticos aos utilizados nas duas etapas anteriores. O último passo da quarta etapa foi somar todos os artigos encontrados até o momento.

\section{temas.}

\section{Quinta etapa: Definição e classificação dos}

Após a seleção dos artigos foi feita a leitura na íntegra, do mais antigo para o mais recente. Como apoio para a leitura dos artigos, dois roteiros foram preenchidos, um que buscava sintetizar o artigo, considerando a introdução, objetivos, procedimentos, resultados e discussão, e outro que buscava identificar os temas abordados e as variáveis dependentes e independentes de cada artigo.

Os temas dos artigos selecionados não foram definidos previamente. Embora para alguns fosse possível identificar tais temas pelo título, resumo e palavras-chave, a leitura na íntegra e o preenchimento de todos os roteiros possibilitou identificar de uma maneira geral quais eram os principais temas. No entanto, para que houvesse uma melhor definição e para que tais temas pudessem ser identificados de forma mais precisa, para cada um deles foi utilizada uma definição conceitual pautada nos princípios da Análise do Comportamento.

Como poderá ser visto na seção de resultados, os três principais temas encontrados foram: comportamento social, autocontrole e metacontingências. As definições utilizadas para cada um desses temas foram:

Comportamento Social - contingência de três termos cujas consequências são mediadas pelo comportamento operante de outro(s) indivíduo(s) (Sampaio \& Andery, 2010);

Autocontrole - escolha que produz reforçamento de maior magnitude e atrasado (Rachlin \& Green, 1972); e

Metacontingência - relação contingente entre: (1) Contingências comportamentais entrelaçadas que produzem um produto agregado e (2) eventos ou condições ambientais selecionadoras (Glenn et al., 2016).

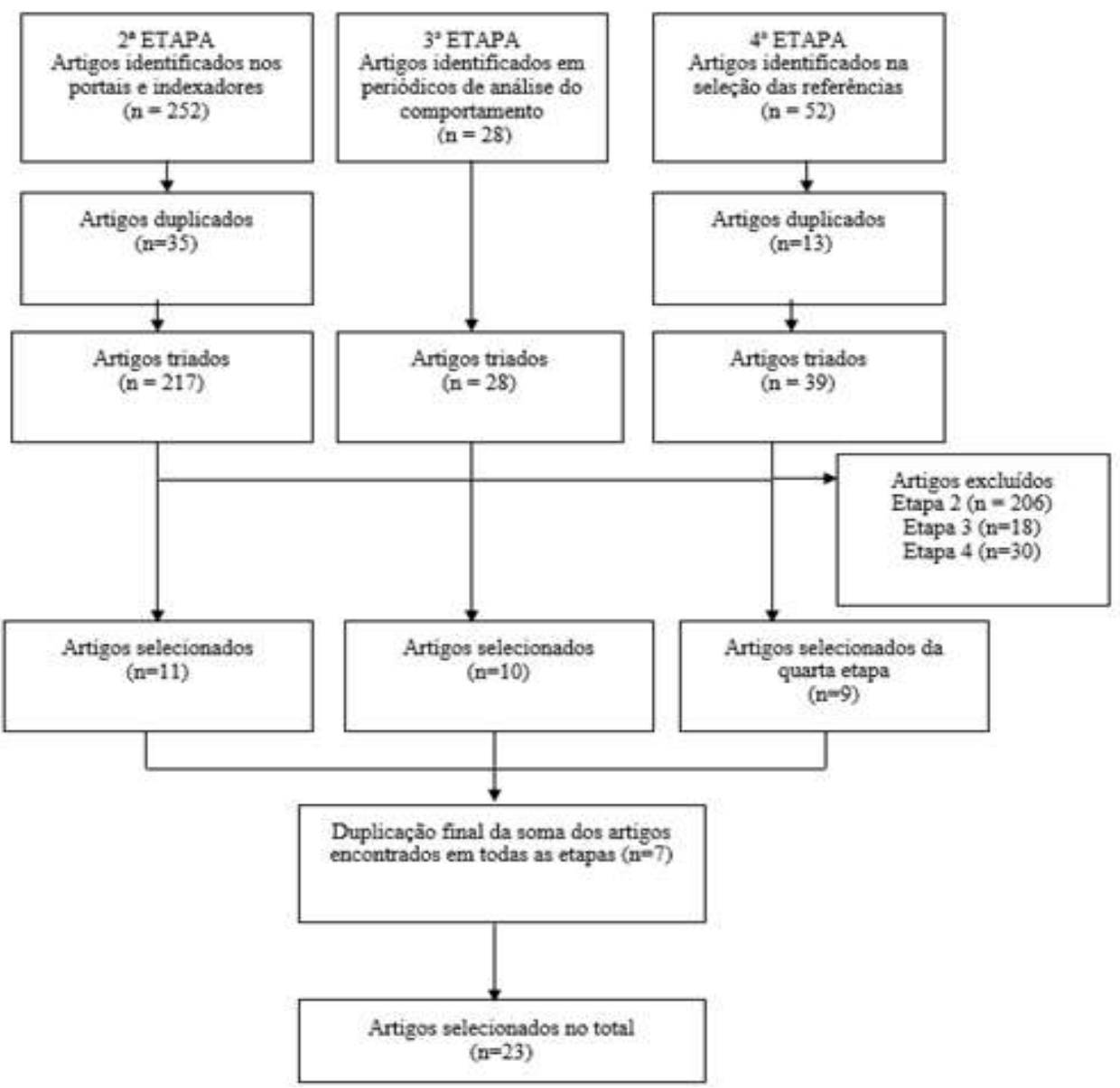

Figura 2. Número de artigos selecionados em cada etapa do procedimento apresentados no Prisma Flow Diagram. ${ }^{4}$

${ }^{4} \mathrm{http} / / /$ www.prisma-statement.org/PRISMAStatement/FlowDiagram.aspx 


\section{RESULTADOS E DISCUSSÃO}

Os resultados foram descritos considerando a quantidade de artigos selecionados e os principais temas relacionados à Análise do Comportamento, nos artigos experimentais que utilizaram como procedimento o Dilema do Prisioneiro.

\section{Artigos selecionados}

Conforme apresentado na Figura 2, foram encontradas 252 referências nos portais e indexadores utilizados para a busca nesse estudo. Desse total, 35 foram excluídas por serem duplicadas, restando 217. A partir da leitura dos resumos e considerando como critérios de seleção o estudo ser experimental, estar pautado na Análise do Comportamento e empregar o Dilema do Prisioneiro na sua metodologia, 206 referências foram excluídas. Apenas 11 artigos, que apresentavam todos os critérios de seleção, foram selecionados na segunda etapa.

$\mathrm{Na}$ terceira etapa, que envolveu a busca nos periódicos disponíveis no site da ABAI, 28 artigos foram encontrados. Desse montante de artigos, somente 10 foram selecionados por apresentarem todos os critérios de seleção.

$\mathrm{Na}$ quarta etapa, foram encontradas 52 novas referências. Desse total, conforme apresentado na Figura 2, 13 artigos foram excluídos por serem duplicados, restando 39 artigos. Com a aplicação dos critérios "ser uma pesquisa experimental", "ser pautado na Análise do Comportamento" e "empregar o Dilema do Prisioneiro na sua metodologia", apenas nove artigos foram selecionados nesta etapa.

Ao final do procedimento, uma nova exclusão de artigos duplicados foi realizada. Dos 30 artigos selecionados no total, sete foram excluídos por serem duplicados, restando apenas 23 artigos selecionados para análise.

\section{Temas dos artigos selecionados}

Conforme apresentado na Figura 3, dos 23 artigos selecionados ao final de todas as etapas da revisão, dez voltaram-se ao estudo do comportamento social, seis para o estudo do autocontrole, quatro envolveram tanto o autocontrole como comportamento social, e três relacionaram o Dilema do Prisioneiro com metacontingências.

Ao longo de quase seis décadas em que a Análise Experimental do Comportamento tem empregado o Dilema do Prisioneiro, a quantidade de temas e de artigos tem aumentado. Até a década de 1990, os experimentos eram realizados somente para investigar variáveis relacionadas ao comportamento social. A partir dessa década, os experimentos que utilizam o Dilema do Prisioneiro passaram a investigar os efeitos de variáveis relacionadas ao autocontrole e também as relações entre esse fenômeno e o comportamento social. Os estudos sobre metacontingências passaram a ser realizados a partir de 2012.

Para todos os temas, inclusive comportamento social, que não teve nenhum artigo publicado nas décadas de 1980 e 1990, a quantidade dos artigos publicados teve um aumento expressivo a partir da década de 2000. A maioria da produção da área ocorreu nas décadas de 2000 e 2010, 15 artigos ao todo, o que equivale a aproximadamente $70 \%$ de toda produção selecionada na revisão. Se ainda for feita uma análise por década, a que tem o maior volume de produção é a de 2010, oito artigos. Como tal década ainda não terminou, pode haver um número ainda maior de artigos produzidos pela Análise Experimental do Comportamento que utilizam o Dilema do Prisioneiro.

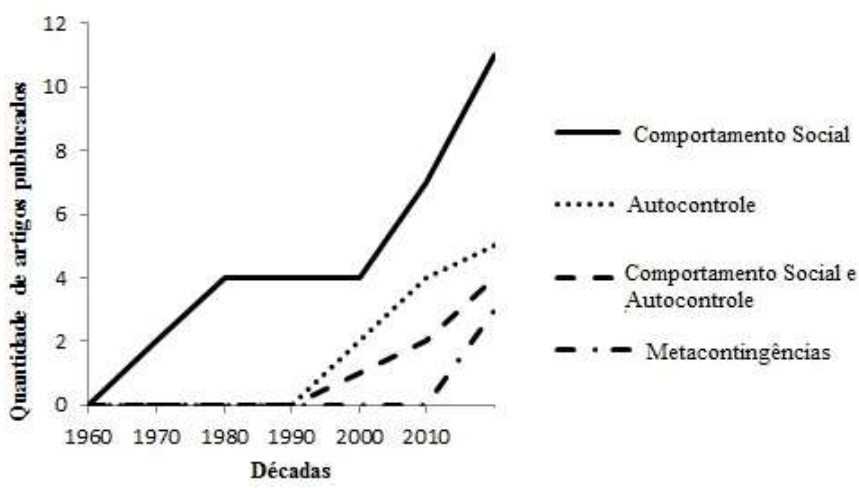

Figura 3. Quantidade de artigos por década, com as curvas cumulativas dos assuntos estudados envolvendo o Dilema do Prisioneiro.

\section{Comportamento social}

Em relação à Análise Experimental do Comportamento e fenômenos relacionados ao comportamento social, Hake e Vulkelich (1972), em uma revisão sobre procedimentos experimentais voltados a investigar a cooperação, identificaram que desde a década de 1940 já havia estudos de laboratório sobre o tema. Esses mesmos estudos se estenderam para as décadas de 1950 e 1960.

Especificamente sobre os estudos que utilizam o Dilema do Prisioneiro com base na Análise Experimental do Comportamento, os primeiros artigos publicados foram realizados por Komorita (1965) e McClintock e McNeel (1966) (Tabela1). Nesse mesmo período, era publicado o livro de Rapoport e Chammah (1965), que apresenta uma compilação de experimentos desenvolvidos por esses autores para investigar variáveis relacionadas ao comportamento social no Dilema do Prisioneiro. Os autores destacam a importância das teorias da aprendizagem na investigação do Dilema do Prisioneiro, especificamente na relação entre estímulos antecedentes, resposta e consequência.

Na década de 1970, o Dilema do Prisioneiro passou a ser um dos modelos mais empregados na investigação de relações sociais, no âmbito da Psicologia Experimental. De acordo com Axelrod (1980), nesta década houve mais de 350 artigos voltados a investigar o Dilema do Prisioneiro citados no Psychological Abstracts. Para este autor a utilização do referido jogo tornou-se uma "epidemia" na Psicologia Social durante os anos 70. No entanto, nessa mesma década foram publicados apenas dois artigos que utilizaram o Dilema do Prisioneiro com 
base na Análise Experimental do Comportamento (Figura 3 e Tabela 1).

As variáveis manipuladas nos artigos que investigaram o comportamento social apresentados na Tabela 1 foram:

1. Interação Verbal (Enzle \& Morrison, 1974), envolvendo a possibilidade dos participantes se comunicarem em determinados momentos durante o jogo;

2. Tipo de feedback (Komorita, 1965), em que eram manipulados tipos de informação ao participante sobre a possibilidade de cooperação ou competição por parte do outro jogador;

3. Falso Feedback (Monterosso, Ainslie, Toppi Mullen, \& Gault, 2002), em que era disponibilizado ao participante um comentário discrepante de qual escolha o outro jogador faria;

4. Presença de outro participante (Jozefowiez \& Darcheville, 2000), que investigava o efeito da presença ou não do outro participante no momento em que as escolhas eram realizadas;

5. Modelo de aprendizagem (Masuda \& Nakamura, 2011), em que foi investigado, em uma simulação computacional, o efeito da aprendizagem prévia na emissão de respostas cooperativas;

6. Desconto social (Ito, Saeki, \& Green, 2011; Locey, Safin, \& Rachlin, 2013), que avalia os efeitos da proximidade e familiaridade entre os participantes sobre as respostas cooperativas;

7. Acesso à pontuação em conjunto com valor da consequência (McClintock \& McNeel, 1966), em que as variáveis independentes eram o acesso à pontuação após cada tentativa no jogo e os valores dos reforçadores, respectivamente;
8. Presença ou ausência do outro jogador e valor da consequência (Schmitt, 1976);

9. Tamanho do grupo, interação verbal e pausa entre tentativas (Yi \& Rachlin, 2004), sendo que esta última variável indicava uma "trégua" durante o jogo com cinco participantes.

Mesmo que haja semelhanças entre os temas dos estudos que utilizaram o Dilema do Prisioneiro com base na Análise Experimental do Comportamento com os estudos pautados na Psicologia Social e Cognitiva (apresentados na introdução), a diferença marcante em todos eles é o fato que os resultados dos primeiros são analisados com base no modelo de seleção do comportamento pelas consequências, considerando que as escolhas feitas pelos participantes ocorrem em função das contingências de reforçamento. Em outra perspectiva, os estudos revisados por Kollok (1998) e Kopelman et al. (2002) analisam as escolhas dos participantes em termos de motivos sociais, fatores perceptuais e mapas cognitivos.

Cabe ressaltar que nos estudos pautados na Análise Experimental do Comportamento, voltados à investigação do comportamento social, as escolhas envolvidas no Dilema do Prisioneiro podem ser definidas como:

(1) Cooperação - escolha mútua entre os jogadores que resulta em um reforço maior para o grupo e que também produz um maior reforçador, ao longo do jogo, individualmente para os participantes;

(2) Competição - escolha entre os jogadores que resulta em um reforço menor para o grupo e também para os jogadores individualmente, quando comparada com o padrão de escolhas cooperativas que poderiam ser obtidas ao final do jogo.

Tabela 1.

Artigos Voltados ao Estudo do Comportamento Social, Autocontrole e Metacontingências, e Suas Respectivas Variáveis Manipuladas. Temas Variáveis manipulas Artigos e variáveis manipuladas

Enzle, M. E., \& Morrison, B. J. (1974). Communication of intentions and requests, and the availability of punitive Power in a mixed-motive situation. Psychological Reports, 34, 8999005 .

Interação verbal

Komorita, S. S. (1965). Cooperative choice in Prisoner`s

Tipo de feedback Dilemma Game. Journal of Personality and Social Psychology, 2, 741-745.

Monterosso, J., Ainslie, G., Toppi Mullen, P., \& Gault, B.

Falso feedback (2002). The fragility of cooperation: A false feedback study of a sequential Iterated prisoner's dilemma, Journal of Economic

Psychology, 23, 437-448.
Jozefowiez, J., \& Darcheville, J.C. (2000). An operant

COMPORTAMENTO SOCIAL
Presença do outro participante

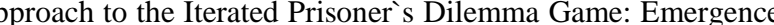
of stable cooperation after indirect reinforcement of controlling behaviors in artificial learning agents. Revista Mexicana de Análisis de laConducta, 26, 2, 211-227.

Masuda, N., \& Nakamura, M. (2011). Numerical analysis of a reinforcement learning model with the dynamic aspiration level in the iterated Prisoner's dilema. Journal of Theoretical Biology. 278, 55-62.

Ito, M., Saeki, D., \& Green, L. (2011). Sharing, discounting, and selfishness: A Japanese-American comparison. The Psychological Record, 60, 59-76.

Desconto social
Locey, M., Safin, V. \& Rachlin, H. (2013). Social discounting and The Prisoner's Dilemma Game. Journal of the Experimental Analysis of Behavior, 99, 85-97. 
Acesso à pontuação e valor da consequência

Presença ou ausência do outro jogador e valor da consequência

Tamanho do grupo; interação verbal e pausa entre tentativas

Atraso do reforço

AUTOCONTROLE

Atraso e valor do reforço.

COMPORTAMENTO SOCIAL E AUTOCONTROLE

\begin{tabular}{c} 
Valor do reforço \\
\hline $\begin{array}{c}\text { Probabilidade de reciprocidade e } \\
\text { Presença de outro participante }\end{array}$ \\
\hline
\end{tabular}

McClintock, C., \& McNeel, S. (1966). Reward and score feedback as determinants of cooperative and competitive game behavior. Journal of Personality and Social Psychology, 4, 606-613.

Schmitt, R. D. (1976). Some conditions affecting the choice to cooperate or compete. Journal of the Experimental Analysis of Behavior, 25, 165-178.

Yi, R., \& Rachlin, H. (2004). Contingencies of reinforcement in a five-person Prisoner`s Dilemma. Journal of the Experimental Analysis of Behavior, 82, 161-176.

Green, L., Price, C.P., \& Hamburger, E.M. (1995). Prisoner`s Dilemma and the pigeon: Control by immediate consequences. Journal of the Experimental Analysis of Behavior, 64, 1. Sanabria, F., Baker, F., \& Rachlin, H. (2003). Learning by pigeons playing against tit-for-tat in an operant prisoner's dilemma. Learning \& Behavior, 31, 4, 318-331.

Yi, R., Buchhalter, R. A., Gatchalian, M. K., \& Bickel, K. W. (2007). The relationship between temporal discounting and the prisoner's dilemma game in intranasal abusers of prescription opioids. Drug and Alcohol Dependence, 87, 94-97.

Reboreda, C. J., \& Kacelnik, A. (1993). The role of autoshaping in cooperative two-player games between starlings. Journal of the Experimental Analysis of Behavior, 60, 67-83.

Yi, R., Johnson, W. M., \& Bickel, K. W. (2005). Relationship between cooperation in an iterated prisoner's dilemma game and the discounting of hypothetical outcomes. Learning \& Behavior, 33, 3, 324-336.

Locey, M., \& Rachlin, H. (2012). Commitment and self-control in a Prisoner`s Dilemma Game. Journal of the Experimental Analysis of Behavior, 98, 89-103.

Silverstein, A., Cross, D., Brown, J., \& Rachlin, H. (1998). Prior experience and patterning in a Prisoner's Dilemma Game. Journal of Behavioral Decision Making, 11, 123-138.

Baker, F., \& Rachlin, H. (2001). Probability of reciprocation in repeated Prisoner's Dilemma Games. Journal of Behavioral Decision Making, 14, 51-67.

Charlton, R. S., Yi, R., Porter, C., Carter, E. A., Bickel, W., \& Rachlin, H. (2013). Now for me, later for us? Effects of group context on temporal discounting. Journal of Behavioral Decision Making, 26, 118-127.

Yi, R., King, F. L., Carter, E. A, Landes, D. R., \& Bickel, K. W. (2010). Intertemporal decision-making for a group. The Psychological Record, 60, 577-586.

Costa, D., Nogueira, V. P. C.. \& Vasconcelos, A. L. (2012).

Effects of communication and cultural consequences on choices combinations in INPDG with four participants. Revista Latinoamericana de Psicologia, 44, 121-131.

Ortu, D., Becker, A., Woelz, T. A. R. \& Glenn, S.S. (2012). An iterated four-player Prisoner`s Dilemma Game with an external selecting agent: A metacontingency experiment. Revista Latinoamericana de Psicologia, 44, 111-120.

Morford, H. Z., \& Cihon, M. T. (2013). Developing an experimental analysis of metacontingencies: Consideration regarding cooperation in a four-person Prisoner`s Dilemma Game. Behavior and Social Issues, 22, 5-20.

\section{Autocontrole}

Segundo Hanna e Todorov (2002), na Análise do Comportamento, Skinner e Rachlin foram grandes influenciadores no desenvolvimento de conhecimentos empíricos, discussões e metodologias sobre o autocontrole. Já em 1953, Skinner discutiu o tema no livro "Ciência e Comportamento Humano". Em 1972, a partir da definição de Rachlin e Green, o autocontrole também passou a ser investigado com base no comportamento de escolha. Para esses autores, autocontrole é compreendido como sendo a escolha de um estímulo que produz uma consequência atrasada e de maior magnitude. A impulsividade, por sua vez, refere-se à escolha de um estímulo que produz um reforço menor e imediato.

Em um estudo de revisão sobre autocontrole, Hanna e Ribeiro (2005) apresentaram as seguintes 
variáveis que têm sido empregadas para investigar tal fenômeno: atraso, probabilidade, frequência, qualidade, magnitude do reforçador e atividades realizadas durante o atraso do reforço. Os artigos encontrados na presente revisão que voltaram-se ao estudo do autocontrole, como apresentado na Tabela 1, manipularam a variável atraso do reforço (Green, Price, \& Hamburger, 1995; Sanabria, Baker, \& Rachlin, 2003; Yi, Buchhalter, Gatchalian, \& Bickel, 2007); conjuntamente atraso e valor do reforço (Reboreda \& Kacelnik, 1993; Yi, Johnson, \& Bickel, 2005) e resposta de comprometimento (Locey \& Rachlin, 2012). O atraso do reforço se refere ao intervalo de tempo entre a resposta e o reforço, enquanto que a magnitude refere-se ao valor do reforço no momento em que o mesmo é disponibilizado (Rachlin \& Green, 1972). As respostas de comprometimento referem-se ao comportamento do participante que dá acesso somente à recompensa maior e atrasada, impossibilitando a escolha da recompensa menor e imediata.

Estudos encontrados na presente revisão de literatura voltados à investigação do autocontrole, pautados na Análise Experimental do Comportamento e que utilizam o Dilema do Prisioneiro Repetido, manipularam os efeitos dos reforços imediatos e de longo prazo no que se refere à escolha do indivíduo (Yi \& Rachlin, 2004).

\section{Comportamento social e autocontrole}

Pesquisas que relacionam comportamento social e autocontrole começaram a ser realizadas com o intuito de identificar como esses fenômenos poderiam influenciar um ao outro (Brown \& Rachlin, 1999), uma vez que ambos já estavam sendo investigados pela área, mas separadamente.

As variáveis manipuladas nos artigos que se voltaram ao estudo tanto do comportamento social quanto do autocontrole (Tabela 1) foram: o desconto temporal e social (Yi, King, Carter, Landes, \& Bickel, 2010; Charlton et al., 2013); o valor do reforço (Silverstein, Cross, Brown, \& Rachlin, 1998); e a presença do outro e a probabilidade de reciprocidade (Baker \& Rachlin, 2001). A variável desconto social envolve a proximidade e familiaridade que os jogadores possuem entre si. Já o desconto temporal refere-se a um procedimento em que o participante do experimento deveria escolher entre duas opções que envolveriam reforços imediatos ou a longo prazo. $\mathrm{O}$ valor do reforço refere-se a ao ganho da pontuação durante o jogo, que é alterada a depender da escolha feita pelo outro participante. A probabilidade de reciprocidade e a presença do outro participante, referem-se respectivamente à probabilidade que $\mathrm{o}$ participante tinha de receber uma resposta recíproca do outro no jogo e a como a presença de outro participante poderia alterar a probabilidade de respostas cooperativas.

Para Brown e Rachlin (1999) o comportamento social e o autocontrole apresentam uma semelhança, pois ambos requererem reciprocidade. O comportamento social requer reciprocidade entre as pessoas e o autocontrole requer reciprocidade de reforçadores para um único indivíduo ao longo do tempo. No ambiente natural esses dois fenômenos podem estar relacionados na maioria das vezes. No comportamento social, a cooperação pode ocorrer em função de um ou pelo menos dois indivíduos abrirem mão de reforçadores no curto prazo para terem acesso a reforçadores de maior magnitude no longo prazo. No caso do autocontrole, mesmo que o indivíduo emita comportamentos controlados ou impulsivos sem a presença física de uma pessoa, seu comportamento estará sob controle de um contexto social. Por exemplo, praticar esportes e ter uma alimentação saudável (autocontrole) podem ser mantidos por uma aprovação social, assim como também o uso de drogas (impulsividade), que pode produzir reforçadores sociais pela comunidade no qual o indivíduo se insere.

Assim como pode ser identificado nos artigos voltados ao estudo do comportamento social e autocontrole, no Dilema do Prisioneiro, ambos os fenômenos podem ser analisados em função da escolha cooperativa, pois essa promove o maior reforçador no longo prazo não só para um único jogador (autocontrole), mas também para todos os participantes do jogo (cooperação).

\section{Metacontingências}

Quando estamos diante de práticas culturais com um determinado nível de complexidade, a contingência de três termos como unidade de análise pode muitas vezes não ser suficiente para analisar tais práticas. Nas práticas culturais, as consequências agem sobre o grupo, produzindo efeitos sobre o mesmo (Andery, Micheletto, \& Sério, 2005).

Glenn, em 1986, utilizou pela primeira vez o conceito de metacontingências. Ao longo dos anos, tal conceito passou por modificações (Martone \& Todorov, 2007). Como já apresentado na seção de método, Glenn et al. (2016) definiram metacontingências como sendo a relação contingente entre: (1) contingências comportamentais entrelaçadas que produzem um produto agregado; e (2) eventos ou condições ambientais selecionadoras.

Vichi (2005) foi o primeiro a demonstrar que havia a possibilidade de estudar em laboratório as práticas culturais utilizando metacontingências como unidade de análise. Toledo (2015) realizou uma revisão da produção de dados experimentais sobre metacontingências e identificou como exemplo, as seguintes variáveis: manipulações de consequências culturais; consequência individual e cultural com naturezas distintas; e substituição de participantes ao longo de "gerações".

A Tabela 1 apresenta os artigos que investigaram as metacontingências relacionadas ao Dilema do Prisioneiro e que manipularam variáveis como: interação verbal e valor da consequência cultural (Costa, Nogueria, \& Vasconcelos, 2012); acesso e valor da consequência cultural (Ortu, Becker, Woelz, \& Gleen, 2012) e; a possibilidade de tirar pontos do parceiro (Morford \& Cihon, 2013).

Em relação às diferentes variáveis manipuladas nos estudos selecionados, a interação verbal, como já mencionada, refere-se à possibilidade de "comunicação" 
entre os participantes durante o jogo. As consequências culturais alteram e mantêm as contingências entrelaçadas (Gleen et al, 2016). A possibilidade de retirar pontos refere-se à oportunidade de o jogador diminuir a quantidade de pontos obtidos pelo outro participante, resultando no aumento dos pontos do primeiro.

Como muitos dos nossos comportamentos são emitidos em grupo, isso envolve a coordenação de comportamentos de múltiplos indivíduos que produzem uma consequência cultural. No caso do Dilema do Prisioneiro, no contexto do grupo, quando os membros optam por qualquer uma das escolhas disponíveis, produzse então o feedback de mercado (consequências culturais), que altera as contingências entrelaçadas (Costa, Nogueira, \& Vasconcelos, 2012).

\section{CONSIDERAÇÕES FINAIS}

Com base nos resultados obtidos foi possível identificar, após a utilização de todos os critérios de busca definidos pelo procedimento, os artigos publicados em periódicos científicos, experimentais, pautados na Análise do Comportamento e que manipularam variáveis, empregando como procedimento o Dilema do Prisioneiro. Dos 332 textos encontrados ao longo do estudo de revisão (identificados na segunda, terceira e quarta etapa), apenas 23 foram selecionados como estudos pautados na Análise Experimental do Comportamento e no Dilema do Prisioneiro - aproximadamente $7 \%$ do total dos artigos encontrados. Cabe ressaltar que esse número deve-se a uma diversidade de áreas que utilizam o Dilema do Prisioneiro para análises e investigações, como a Biologia, Economia, Ciência Política e Relações Internacionais.

Ainda em relação ao número de artigos inicialmente encontrados no presente estudo, é importante ressaltar que muitos eram teóricos, conceituais, pesquisas observacionais, longitudinais e transversais. Além disso, também foram encontradas dissertações, teses, livros, capítulos de livros e resumos de anais de eventos científicos, que não eram os tipos de textos que faziam parte dos critérios de seleção e por isso foram excluídos.

Também cabe destacar que nenhum artigo selecionado foi publicado em um periódico brasileiro, o que sugere que no Brasil, pouca pesquisa na área tem sido feita e/ou não tem sido publicada no país. Dois dos artigos sobre metacontingências tinham como autores pesquisadores brasileiros (Costa, Nogueira, \& Vasconcelos, 2012; Ortu, Becker, Woelz, \& Glenn, 2012), porém a publicação foi feita em língua inglesa em um mesmo periódico estrangeiro.

Em relação à metodologia, um aspecto relevante é o fato de que ao meramente inserir palavras em campos de busca de indexadores ou portais, não necessariamente todos os artigos que estão cadastrados nos mesmos poderiam ser encontrados. Além disso, se as palavras definidas na seleção dos artigos mencionados na seção de referências dos mesmos até então encontrados fossem inseridas nos campos de busca, já na primeira etapa, possivelmente a quantidade de estudos selecionados inicialmente seria maior.
Ainda é importante lembrar que os artigos lidos eram de periódicos que disponibilizam os textos gratuitamente, via Portal de Periódicos da Capes, com convênio pela instituição de ensino em que a revisão foi realizada. Se a pesquisa dos artigos fosse estendida para periódicos em que o acesso aos mesmos é disponibilizado apenas por pagamento, outros experimentos poderiam ter sido encontrados e compor o número de artigos selecionados.

Com base nos dados obtidos é possível afirmar que a Análise Experimental do Comportamento tem ampliado o escopo de investigação relacionado ao Dilema do Prisioneiro. Em primeiro lugar, no âmbito do comportamento social, os experimentos identificados destacaram o papel das contingências de reforçamento e especificamente os efeitos das condições antecedentes e das consequências na aquisição e manutenção de respostas de cooperação e competição. Em segundo lugar, em relação às pesquisas sobre autocontrole, a Análise Experimental do Comportamento é pioneira em investigar as variáveis relacionadas a esse fenômeno comportamental, utilizando o referido jogo. Em terceiro lugar, a contribuição em relação a esse segundo aspecto é ampliada quando os fenômenos sociais são investigados na sua relação com o autocontrole. Essa temática de investigação pode produzir um conhecimento de extrema relevância, não só nos estudos envolvendo o Dilema do Prisioneiro, mas na Psicologia em geral, como também em outras áreas do conhecimento.

Por fim, no contexto de investigação das metacontingências, o Dilema do Prisioneiro pode ser utilizado para análise do desenvolvimento, manutenção e transmissão de contingências culturais entrelaçadas e os seus produtos. Em função do aumento, nas últimas duas décadas, dos estudos experimentais sobre metacontingências, de um modo geral e também daqueles que relacionam tal unidade de análise com o Dilema do Prisioneiro, essa parece ser uma das linhas de investigação que mais tende a se desenvolver nos próximos anos. No entanto, os estudos que utilizam o Dilema do Prisioneiro, pautados na Análise Experimental do Comportamento também, muito provavelmente, continuarão a ser empregados para a análise do comportamento social e autocontrolado.

Os dados produzidos nesta revisão podem permitir aos leitores uma visão abrangente dos artigos selecionados por utilizarem como procedimento o Dilema do Prisioneiro, pautados na Análise Experimental do Comportamento. Além disso, a presente revisão possibilita o conhecimento das variáveis manipuladas e dos fenômenos investigados (comportamento social, autocontrole e metacontingências). Entretanto, o resultado obtido apresenta apenas uma parcela da produção na Análise do Comportamento. Futuros estudos de revisão sistemática de literatura poderiam investigar outros textos além de artigos experimentais, como artigos teóricos e/ou conceituais, dissertações e teses, permitindo a ampliação do conhecimento dos estudos relacionados na área. 


\section{REFERÊNCIAS $^{5}$}

Alarcão, I., Cardoso, T., \& Celorico, A. J. (2010). Revisão da literatura e sistematização do conhecimento. Porto: Porto Editora.

American Psychological Association. (2010). Publication manual of the American Psychological Association (6th ed.). Washington, DC: Author.

Andery, M. A. P. A., Micheletto, N., \& Sério, T. M. A. (2005). A análise de fenômenos sociais: Esboçando uma proposta para a identificação de contingências entrelaçadas e metacontingências. Revista Brasileira de Análise do Comportamento, 1 (2), 149-165. http://dx.doi.org/10.18542/rebac.v1i2.2167

Axelrod, R. (1980). Effective choice in the Prisoner's Dilemma. Journal of Conflict Resolution, 24 (1), 3-25. https://doi.org/10.1177/002200278002400101

*Baker, F. \& Rachlin, H. (2001). Probability of reciprocation in repeated Prisoner's Dilemma Games. Journal of Behavioral Decision Making, 14 (1), 51-67. doi:10.1002/1099-0771(200101)14:1<51::AIDBDM365>3.0.CO;2-K.

Brown, J. \& Rachlin, H. (1999) Self-control and social cooperation. Behavioral Processes, 47 (2), 65-72. doi 10.1016/S0376-6357(99)00054-6.

*Charlton, R. S., Yi, R., Porter, C., Carter, E. A., Bickel, W., \& Rachlin, H. (2013). Now for Me, Later for Us? Effects of group context on temporal discounting. Journal of Behavioral Decision Making, 26, 118-127. doi: $10.1002 / b d m .766$

*Costa, D., Nogueira, C. de P. V., \& Vasconcelos, L. A. (2012). Effects of communication and cultural consequences on choices combinations in INPDG with four participants. Revista Latino americana de Psicología, 44(1), 121-131. Recuperado de http://www.scielo.org.co/scielo.php?script=sci_arttext \&pid=S0120-05342012000100012\&lng=pt\&tlng=en.

Dawes, R. B. (1980). Social dilemmas. Annual Review Psychology, 31, 169-193. doi: 10.1146/annurev.ps.31.020180.001125

*Enzle, M. E., \& Morrison, B. J. (1974). Communication of intentions and requests, and the availability of punitive Power in a mixed-motive situation. Psychological Reports, 34, 899-9005. https://doi.org/10.2466/pr0.1974.34.3.899

Glenn, S. S., Malott, M. E, Andery, M. A. P. A., Benvenuti, M., Houmanfar, R. A., Sandaker, I., Todorov, J. C., Tourinho, E., \& Vasconcelos, L. A. (2016). Toward consistent terminology in a behaviorist approach to cultural analysis. Behavior and Social Issues, $\quad 25, \quad 11-27$. http://dx.doi.org/10.5210/bsi.v25i0.6634

*Green, L., Price, C.P., \& Hamburger, E.M. (1995). Prisoner`s Dilemma and the pigeon: Control by immediate consequences. Journal of the Experimental

\footnotetext{
${ }^{5}$ As referências que apresentam um asterisco (*) são aquelas que foram encontradas na revisão do presente manuscrito e que, portanto, descrevem experimentos pautados na Análise do Comportamento e que o utilizam o Dilema do Prisioneiro.
}

Analysis of Behavior, 64 (1), 1-17. doi: 10.1901/jeab.1995.64-1

Hake, D. F., \& Vukelick, R. (1972). A classification and review of cooperation procedures. Journal of the Experimental Analysis of Behavior, 18 (2), 333-343. doi: 10.1901/jeab.1972.18-333

Hanna, E. S., \& Ribeiro, M. R. (2005). Autocontrole: Um caso especial de comportamento de escolha. In J. Abreu-Rodrigues \& M. R. Ribeiro (Orgs.), Análise do Comportamento: Pesquisa, Teoria e Aplicação (pp. 175-1870). São Paulo: ARTMED.

Hanna, E. S., \& Todorov, J. C. (2002). Modelos de autocontrole na análise experimental do comportamento: Utilidade e crítica. Psicologia: Teoria e Pesquisa, 18(3), 337343. https://dx.doi.org/10.1590/S010237722002000300014

*Ito, M., Saeki, D, \& Green, L. (2011). Sharing, discounting, and selfishness: A Japanese-American comparison. The Psychological Record, 60, 59-76. Recuperado http://opensiuc.lib.siu.edu/cgi/viewcontent.cgi?article= $1378 \&$ context $=$ tpr

*Jozefowiez, J., \& Darcheville, J.C. (2000) An operant approach to the Iterated Prisoner's Dilemma Game: Emergence of stable cooperation after indirect reinforcement of controlling behaviors in artificial learning agents. Revista Mexicana de Análisis de la Conducta, 26, 211-227. Recuperado de http://rmacmx.org/wp-content/uploads/2013/05/Vol-26-n-2-211227.pdf

Kollock, P. (1998) Social dilemmas: The anatomy of cooperation. Annual Review of Sociology, 24, 183-214. Recuperado de http://www.rheingold.com/texts/KollockSocialDilemm as.pdf

Komorita, S. S. (1965). Cooperative choice in Prisoner's Dilemma Game. Journal of Personality and Social $\begin{array}{llll}\text { Psychology, } & 2 & \text { (5), }\end{array}$ http://dx.doi.org/10.1037/h0022673

Kopelman, S., Weber, J. M., \& Messick, D. M. (2002). Factors influencing cooperation in commons dilemmas: A review of experimental psychological research. In: E. Ostrom, T. Dietz, N. Dolsak, P. C. Stern, S. Stonich, \& E. U. Weber (Eds.). The Drama of the Commons. (pp.113-156). National Academy Press: Washington, DC. Disponível em: http://books.nap.edu/catalog.php?record_id=10287\#to $c$.

*Locey, M., \& Rachlin, H. (2012). Commitment and selfcontrol in a Prisoner`s Dilemma Game. Journal of the Experimental Analysis of Behavior, 98, 89-103. doi 10.1901/jeab.2012.98-89

*Locey, M., Safin, V., \& Rachlin, H. (2013). Social discounting and The Prisoner`s Dilemma Game. Journal of the Experimental Analysis of Behavior. 99, 85-97. doi: 10.1002/jeab.3

Martone, R. C., \& Todorov, J. C. (2007). O desenvolvimento do conceito de metacontingência. 
Revista Brasileira de Análise do Comportamento, 3, 181-190. Recuperado de http://periodicos.ufpa.br/index.php/rebac/article/viewFi le/830/1177

*Masuda, N., \& Nakamura, M. (2011). Numerical analysis of a reinforcement learning model with the dynamic aspiration level in the iterated Prisoner's dilemma. Journal of Theoretical Biology, 278, 55-62. https://doi.org/10.1016/j.jtbi.2011.03.005

*McClintock, C. \& McNeel, S. (1966). Reward and score feedback as determinants of cooperative and competitive game behavior. Journal of Personality and Social Psychology, 4 (6), 606-613. http://dx.doi.org/10.1037/h0023986

*Monterosso, J., Ainslie, G., Toppi Mullen, P., \& Gault, B. (2002). The fragility of cooperation: A false feedback study of a sequential Iterated prisoner's dilemma, Journal of Economic Psychology, 23, 437448. doi 10.1016/S0167-4870(02)00095-8

*Morford, H. Z., \& Cihon, M. T. (2013). Developing an experimental analysis of metacontingencies: Consideration regarding cooperation in a four-person Prisoner`s Dilemma Game. Behavior and Social Issues, 22, 5-20. http://dx.doi.org/10.5210/bsi.v22i0.4207

*Ortu, D., Becker, A., Woelz, T. A. R., \& Glenn, S. S. (2012). An iterated four-player Prisoner`s Dilemma Game with an external selecting agent: A metacontingency experiment. Revista Latinoamericana de Psicologia, 44,1, 111-120. Recuperado de http://www.scielo.org.co/pdf/rlps/v44n1/v44n1a11.pdf

Poundstone, W. (1992). Prisoner's Dilemma. New York: Doubleday.

Rachlin, H., Brown, J., \& Baker, F. (2001). Reinforcement and punishment in the Prisoner's Dilemma Game. In D. L. Medin (Ed.), The psychology of learning and motivation: Advances in research and theory (pp. 327364). San Diego, CA: Academic Press.

Rachlin, H., \& Green, L. (1972). Commitment, choice and self-control. Journal of the Experimental Analysis of Behavior, $17 \quad$ (1), 15-22. http://doi.org/10.1901/jeab.1972.17-15

Rapoport, A., \& Chammah, A. M. (1965). Prisoner's Dilemma: A study in conflict and cooperation. Ann Arbor: The University of Michigan Press.

*Reboreda, J. C., \& Kacelnik, A. (1993). The role of autoshaping in cooperative two-player games between starlings. Journal of the Experimental Analysis of Behavior, 60, 67-83. doi:10.1901/jeab.1993.60-67

Sampaio, A. A. S., \& Andery, M. A. P. A. (2010). Comportamento social, produção agregada e prática cultural: Uma análise comportamental de fenômenos sociais. Psicologia: Teoria e Pesquisa, 26, 183-192. https://dx.doi.org/10.1590/S0102-37722010000100020

*Sanabria, F., Baker, F., \& Rachlin, H. (2003). Learning by pigeons playing against tit-for-tat in an operant prisoner's dilemma. Learning \& Behavior, 31, 318331. doi: 10.3758/BF03195994

*Schmitt, R. D. (1976). Some conditions affecting the choice to cooperate or compete. Journal of the Experimental Analysis of Behavior, 25, 165-178. doi: 10.1901/jeab.1976.25-165

*Silverstein, A., Cross, D., Brown, J., \& Rachlin, H. (1998). Prior experience and patterning in a Prisoner's Dilemma Game. Journal of Behavioral Decision Making, 11, 123-138. doi:10.1002/(SICI)10990771(199806)11:2<123::AID-BDM283>3.0.CO;2-5

Skinner, B. F. (1953/2000). Ciência e comportamento humano. São Paulo: Martins Fontes.

Toledo, T. F. N. (2015). Debatendo a noção de metacontingência a partir da revisão e produção de dados experimentais. Tese de Doutorado, Instituto de Psicologia da Universidade de São Paulo, São Paulo. Recuperado de http://www.teses.usp.br/teses/disponiveis/47/47132/tde $-04042016-151350$

Vichi, C. (2005). Igualdade ou desigualdade: manipulando um análogo experimental de prática cultural em laboratório. In J. C. Todorov, R. C. Martone, \& M. B. Moreira (Orgs.), Metacontingências: Comportamento, cultura e sociedade (pp.86-100). Santo André, SP: ESETec.

*Yi, R., Buchhalter, R. A., Gatchalian, M. K., \& Bickel, K. W. (2007). The relationship between temporal discounting and the prisoner's dilemma game in intranasal abusers of prescription opioids. Drug and Alcohol Dependence, 87, 94-97. doi:10.1016/j.drugalcdep.2006.07.007

*Yi, R., Johnson, W. M., \& Bickel, K. W. (2005). Relationship between cooperation in an iterated prisoner's dilemma game and the discounting of hypothetical outcomes. Learning \& Behavior, 33, 324336. doi: 10.3758/BF03192861

*Yi, R., King, F. L., Carter, E.A, Landes, D.R., \& Bickel, K.W. (2010). Intertemporal decision-making for a group. The Psychological Record, 60, 577-586. Recuperado de https://www.ncbi.nlm.nih.gov/pmc/articles/PMC42956 30/pdf/nihms602578.pdf

*Yi, R., \& Rachlin, H. (2004). Contingencies of reinforcement in a five-person Prisoner's Dilemma. Journal of the Experimental Analysis of Behavior, 82, 161-176. http://doi.org/10.1901/jeab.2004.82-161

Zamboni, A. B., Thommazo, A. D., Hernandes, E. C. M., \& Fabbri, S. C. P. F. (2010). Start ${ }^{\circledR}$ uma ferramenta computacional de apoio à revisão sistemática. In: CBSoft - Congresso Brasileiro de Software, Salvador BA. CBSoft - Congresso Brasileiro de Software.

Submetido em 21/01/2017 Aceito em 16/08/2017 